ORIGINAL ARTICLE

\title{
Perinatal and postnatal factors in very preterm infants and subsequent cognitive and motor abilities
}

\section{R W I Cooke}

Arch Dis Child Fetal Neonatal Ed 2005;90:F60-F63. doi: 10.1136/adc.2004.059188

See end of article for author's affiliation ......................

Correspondence to: Professor Cooke, Department of Child Health, University of Liverpool, Neonatal Unit, Liverpool Maternity Hospital, Liverpool 18 7SS, UK; mc19@liv.ac.uk

Accepted 8 September 2004

\begin{abstract}
Background: Many children born very preterm have cognitive and minor motor problems later, even if attending mainstream schools.

Objective: To examine associations between this suboptimal performance and perinatal and postnatal clinical factors.

Participants and methods: A geographically determined cohort of 280 infants born at less than 32 weeks gestation in 1991-92 in Liverpool, free of major disability and attending mainstream school at 7 years of age. A perinatal and postnatal data set were obtained from the clinical records retrospectively. Measurements of height, weight, and head circumference at 7 years were made, together with assessments of intelligence (Wechsler intelligence scale for children, IQ) and motor impairment (Movement assessment battery for children, MABC).

Results: Multiple regression analysis showed $I Q$ at 7 years to be independently significantly related to gestation, persistence of the arterial duct (PDA), and head circumference at 7 years. MABC was only significantly related to gestation. PDA was related to periventricular haemorrhage (OR 2.7, 95\% confidence interval (CI) 1.3 to 5.8 ) and parenchymal lesions including ventriculomegaly (OR $4.1,95 \% \mathrm{Cl}$ 1.5 to 11.4$)$.

Conclusion: Cognitive ability in children born preterm is significantly related to gestation, but may also be related to the effects of PDA on early brain development, through either periventricular haemorrhage/ ventriculomegaly or other disturbance of early brain growth.
\end{abstract}

B etween $10 \%$ and $15 \%$ of very preterm infants have subsequent major disabilities in childhood. ${ }^{1}$ Of those attending mainstream schools, up to $40 \%$ have behavioural, motor or learning difficulties. ${ }^{2-4}$ Although major disabilities are predicted by variables describing a stormy neonatal course such as duration of ventilation, cerebral haemorrhage, and seizures, lesser disabilities in those at mainstream schools are not. ${ }^{5}$ The latter have been shown to be related to lower gestational age, suboptimal postnatal growth patterns, and social factors. ${ }^{67}$

This study aimed to examine the associations between perinatal and postnatal factors and cognitive and motor abilities at age 7 years in a geographically selected cohort of very preterm infants born over a two year period, in a period of modern perinatal care practices. They were free of major neurodisability and attending mainstream schools.

\section{METHODS \\ Participants}

All infants born before 32 completed weeks during 1991-92 in the eight hospitals that then existed in the Liverpool postal districts were ascertained. Those who died before discharge from hospital or whose mothers were not resident within a Liverpool postal district at the time of birth were excluded. After the family doctor had been contacted to check on current health status and school placement, the child's parents were approached to seek consent to take part in the study. Children were mainly tested at their schools, although a few parents requested testing at another location. The study protocol was approved by the local research ethics committee, and informed written consent given by the parents.

\section{Tests and measurements}

Perinatal data were obtained from the hospital records by a clerical research assistant using a standard proforma.
Definitions for the variables collected are given in the Appendix. Measurements of the child's height, weight, and head circumference were obtained in a standard manner at the time of testing, and recorded on " 4 in 1 " growth charts (Growth Foundation) for boys and girls, to obtain age and sex appropriate centiles. Intelligence quotient (IQ) was measured using the Wechsler intelligence scale for children $\mathrm{UK}^{8}$ (WISC III) by three research assistants trained in its use. Fine and gross motor skills were assessed using age band 2 of the Movement assessment battery for children (MABC). ${ }^{9}$ Eight items test manual dexterity, ball skills, and static and dynamic balance, and each are scored from 0 to 5 . Higher scores indicate greater impairment. Scores of more than 14/ 40 are below the 5 th centile and are considered to indicate significant disability, and scores of 10-14/40 are considered to indicate "borderline" difficulties.

Twenty perinatal and postnatal variables were compared according to whether the IQ was above or below the mean for the group and whether the MABC score was above or below the 5th centile. Unpaired $t$ tests or Mann-Whitney U tests were used for continuous data, and the $\chi^{2}$ test for categorical data. The IQ cut point was selected as the mean, as very few of the children in mainstream school had an IQ 2 SDs below the mean. The MABC cut point at the 5th centile has been used as a definition of developmental coordination disorder by other authors. Variables that differed significantly $(\mathrm{p}<0.01)$ between the higher and lower IQ and MABC score groups were entered into multiple regression analyses as independent variables with full IQ and MABC scores as the dependent variables respectively. The significance level used served to limit the number of variables entered into the regression model. The multiple regression analyses were

Abbreviations: IQ, intelligence quotient; $M A B C$, Movement assessment battery for children; PDA, patent ductus arteriosus 
Table 1 Univariate analysis of perinatal and postnatal variables according to intelligence quotient (IQ) (above and below mean)

\begin{tabular}{llll}
\hline Variable & IQ $\geqslant 89$ & $I Q<89$ & p Value \\
\hline Gestation (weeks) & $30.2(1.7)$ & $29.3(2.5)$ & 0.001 \\
Birthweight centile† & $50(25-75)$ & $50(25-75)$ & 0.68 \\
Birth OFC centile† & $50(25-75)$ & $50(25-75)$ & 0.91 \\
Maternal illness & $17 / 132(13 \%)$ & $7 / 125(6 \%)$ & 0.045 \\
Pregnancy hypertension & $20 / 132(15 \%)$ & $18 / 126(14 \%)$ & 0.84 \\
PROM & $40 / 132(30 \%)$ & $44 / 126(35 \%)$ & 0.43 \\
APH & $20 / 132(15 \%)$ & $25 / 126(20 \%)$ & 0.32 \\
Antenatal steroids & $45 / 132(34 \%)$ & $41 / 126(33 \%)$ & 0.79 \\
Caesarean section & $67 / 132(51 \%)$ & $57 / 126(45 \%)$ & 0.37 \\
Days ventilation† & $0(0-4)$ & $1.5(0-7)$ & 0.003 \\
Days added oxygen† & $1(0-6)$ & $2(0-18)$ & 0.011 \\
Sepsis & $57 / 132(43 \%)$ & $62 / 124(50 \%)$ & 0.27 \\
PDA & $8 / 132(6 \%)$ & $30 / 124(24 \%)$ & 0.000 \\
Pneumothorax & $6 / 132(5 \%)$ & $12 / 124(10 \%)$ & 0.108 \\
Hypoglycaemia & $18 / 132(14 \%)$ & $15 / 124(12 \%)$ & 0.71 \\
PVH & $20 / 132(15 \%)$ & $26 / 124(21 \%)$ & 0.19 \\
Parenchymal lesion & $3 / 132(2 \%)$ & $15 / 124(12 \%)$ & 0.002 \\
Height 7 years, centile† & $25(9-50)$ & $25(9-50)$ & 0.14 \\
OFC 7 years, centile† & $9(2-50)$ & $9(0.4-25)$ & 0.006 \\
Weight 7 years, centile† & $50(25-75)$ & $25(9-75)$ & 0.047 \\
\hline *Values are mean (SD). & & & \\
†Values are mean (interquartile range). & & \\
OFC, Occipitofrontal circumference; PROM, premature rupture of the membranes; APH, antepartum \\
haemorrhage; PDA, patent ductus arteriosus; PVH, periventricular haemorrhage. & \\
\hline
\end{tabular}

repeated omitting variables that no longer reached significance $(\mathrm{p}<0.05)$.

\section{RESULTS}

A total of 382 children were identified as having been born in the area and period specified. Thirty three had moved away or could not be traced, 18 had died after discharge from hospital, 29 were attending special schools, 16 parents declined to take part, and six failed to attend for testing. This left 280 children who were tested at a mean age of 89.8 months (range $82-101$ ). There were 151 boys (53.3\%). There were 215 singletons, 56 twins, and nine triplets. Thirteen children were tested at the Institute of Child Health, Liverpool, and one at home at the parent's request or because of school holidays.
Mean (SD) total IQ was 89.4 (14.2) and median (interquartile range) MABC score was 8.5 (3.1-15.4). Eighty (29\%) had an MABC score below the 5th centile.

Mean (SD) weight was 23.9 (4.5) $\mathrm{kg}$ for boys and 24.4 (4.8) $\mathrm{kg}$ for girls. Mean (SD) height was 121.8 (5.5) $\mathrm{cm}$ for boys and $121.5(6.1) \mathrm{cm}$ for girls. Mean (SD) head circumference was $51.7(1.8) \mathrm{cm}$ for boys and $51.2(1.8) \mathrm{cm}$ for girls.

Tables 1 and 2 show the values for the perinatal and postnatal variables determined according to IQ and MABC score respectively. In $5.7 \%$ of cases, one or more data points were missing. On univariate analysis, an IQ below the mean for the group was significantly associated $(p<0.01)$ with shorter gestation, more days of ventilation and oxygen therapy, patent ductus arteriosus (PDA), a parenchymal

Table 2 Univariate analysis of perinatal and postnatal variables according to Movement assessment battery for children (MABC) scores (above and below 5th centile)

\begin{tabular}{llll}
\hline Variable & MABC $\leqslant 14$ & MABC $>14$ & p Value \\
\hline Gestation (weeks) & $30.0(2.0)$ & $29.4(2.5)$ & 0.03 \\
Birthweight centile† & $50(25-75)$ & $50(25-75)$ & 0.37 \\
Birth OFC centile† & $50(25-75)$ & $50(17-75)$ & 0.38 \\
Maternal illness & $17 / 189(9 \%)$ & $8 / 77(10 \%)$ & 0.72 \\
Pregnancy hypertension & $24 / 189(13 \%)$ & $15 / 78(19 \%)$ & 0.17 \\
PROM & $58 / 189(31 \%)$ & $29 / 78(37 \%)$ & 0.30 \\
APH & $35 / 189(19 \%)$ & $14 / 78(18 \%)$ & 0.91 \\
Antenatal steroids & $68 / 189(36 \%)$ & $19 / 78(24 \%)$ & 0.06 \\
Caesarean section & $91 / 189(48 \%)$ & $39 / 78(50 \%)$ & 0.78 \\
Days ventilation† & $0(0-5)$ & $1(0-5)$ & 0.23 \\
Days added oxygen† & $1(0-6)$ & $2(0-9.5)$ & 0.06 \\
Sepsis & $85 / 188(45 \%)$ & $36 / 77(47 \%)$ & 0.82 \\
PDA & $24 / 188(13 \%)$ & $16 / 77(21 \%)$ & 0.10 \\
Pneumothorax & $12 / 188(6 \%)$ & $6 / 77(8 \%)$ & 0.68 \\
Hypoglycaemia & $21 / 188(11 \%)$ & $14 / 77(18 \%)$ & 0.13 \\
PVH & $36 / 188(19 \%)$ & $11 / 77(14 \%)$ & 0.46 \\
Parenchymal lesion & $9 / 188(5 \%)$ & $9 / 77(12 \%)$ & 0.65 \\
Height 7 years, centile† & $25(9-50)$ & $25(2-50)$ & 0.43 \\
OFC 7 years, centile† & $9(2-50)$ & $9(0.4-25)$ & 0.26 \\
Weight 7 years, centile† & $25(9-75)$ & $25(9-75)$ & 0.45 \\
\hline
\end{tabular}

*Values are mean (SD)

†Values are mean (interquartile range).

OFC, Occipitofrontal circumference; PROM, premature rupture of the membranes; APH, antepartum haemorrhage; PDA, patent ductus arteriosus; PVH, periventricular haemorrhage. 
Table 3 Final multiple regression analysis summary, with intelligence quotient as dependent variable

\begin{tabular}{lllll}
\hline Variable & B & Standard error & p Value & OR (95\% Cl) \\
\hline Constant & 46.8 & 12.2 & 0.000 & \\
Gestation (weeks) & 1.4 & 0.41 & 0.001 & 4.0 (1.8 to 9.2) \\
PDA & -7.84 & 2.4 & 0.001 & $3.9(3.2$ to 20) \\
OFC 7 year centile & 0.06 & 0.03 & 0.041 & 1.06 (0 to 1.1$)$ \\
\hline OFC, Occipitofrontal circumference; PDA, patent ductus arteriosus.
\end{tabular}

brain lesion on ultrasound scan, and lower head circumference at time of testing (table 1). An MABC score below the 5 th centile was only significantly related to lower gestation $(\mathrm{p}=0.03)$ (table 2).

When the final model was considered, the multiple regression analysis for IQ showed that gestational age, PDA, and head circumference centile at 7 years were independently significantly related to IQ (table 3 ).

\section{DISCUSSION}

Intelligence at school age in children born very preterm appears to be best predicted in this study by gestational age, present head circumference, and PDA in the neonatal period. Gestational age at birth has been associated with almost all developmental outcomes in reported studies, and particularly IQ. ${ }^{10}$ Including gestation in analyses such as these is a problem, as many variables related to maturity that may be explanatory for later IQ and are highly collinear with gestation are removed from the model as being no longer significant. The decreasing mean IQ with lower gestations may simply be related to greater illness severity, or possibly the effect of a disturbance in brain development from earlier birth. Gestation was strongly related to IQ (OR 4, 95\% CI 1.8 to 9.2 for each week) The odds ratio for a later IQ below the mean if there was a PDA in the neonatal period was 3.9 (95\% CI 3.2 to 20). This was independent of gestation and occipitofrontal circumference at 7 years (table 3 ).

Persistence of the arterial duct after birth is a common phenomenon in the preterm infant, and is associated with a variety of acute and longer term complications such as pulmonary haemorrhage and chronic lung disease. ${ }^{11}$ Therapeutic closure of the duct either surgically or pharmacologically may produce short term benefits such as improvement in lung compliance, but has not shown improvements in longer term outcomes in clinical trials. ${ }^{12}$ Larger PDA is associated with hypotension and low cerebral blood flow in the early postnatal period ${ }^{13}$ and an increased risk of periventricular haemorrhage. ${ }^{14}$ It is possible that the explanation for the association seen in this study between PDA and later IQ lies in the relation of PDA to periventricular haemorrhage (OR 2.7, 95\% CI 1.3 to 5.8 ) and parenchymal lesions seen on ultrasonography such as ventriculomegaly (OR 4.1, 95\% CI 1.5 to 11.4), even though these children did not show overt signs of neurological sequelae such as cerebral palsy. Inclusion of preterm birth with major neurodevelopmental sequelae in this study may well have increased the influence of PDA on outcome because of these associations.

Follow up studies of prophylaxis of PDA using indomethacin have not shown differences in developmental outcomes at 2 years. ${ }^{15}$ This could be simply that 2 years of age is too early to detect later differences in IQ, or that the effect of PDA on the preterm brain occurs very early, or despite indomethacin prophylaxis. Alternatively, PDA could be a surrogate for some other aspect of immaturity in the infant related to early brain development. Certainly the infants who did less well had greater ventilatory requirements and spent longer in added oxygen, which could have been as the result of PDA.

The degree of association of PDA with IQ was not seen with MABC scores, where only gestation appeared significant. This would tend to suggest that the determinants of the two functions are different. Recent magnetic resonance imaging studies of school children born preterm showed changes in caudate and hippocampal volumes to be associated with low $\mathrm{IQ}$, but differences in cortical white matter T2 relaxation times (possibly related to myelination) to be associated with greater impairment on MABC scores. ${ }^{16}{ }^{17}$ Although both of these changes are probably related to postneonatal growth patterns, they may have been triggered by brief perinatal insults such as PDA, with or without subsequent periventricular haemorrhage/periventricular leucomalacia or ventriculomegaly. The mechanism by which PDA affects the brain is probably hypotension and reduced cerebral blood flow caused by large ductal flows out of the systemic circulation in the face of a failing immature myocardium. Improved supportive management during this critical period may optimise long term cognitive abilities in this high risk group.

\section{Author's affiliation}

R W I Cooke, Department of Child Health, University of Liverpool, Neonatal Unit, Liverpool Maternity Hospital, Liverpool, UK

Competing interests: none declared

\section{APPENDIX}

\section{DESCRIPTION OF VARIABLES USED IN THE ANALYSIS}

Centiles. For birth weight and head circumferences derived from Growth Foundation charts.

Maternal illness. Any chronic illness likely to affect pregnancy outcome-for example, diabetes, renal failure, systemic lupus, etc.

Pregnancy induced hypertension. Hypertension of a degree that involved obstetric or medical intervention.

Prolonged rupture of amniotic membranes. At least 24 hours before delivery.

Antepartum haemorrhage. Bleeding of more than $500 \mathrm{ml}$ before the onset of labour.

Antenatal steroids. Any course of prophylactic steroids given, complete or otherwise.

Caesarean section. Elective or emergency caesarean section.

Sepsis. Positive blood culture in newborn.

Persistent arterial duct. Clinical diagnosis leading to a change in management. Usually but not always confirmed on echocardiography.

Pneumothorax. Treatment with chest drain.

Hypoglycaemia. Any occurrence resulting in a change in management.

Periventricular haemorrhage. Evidence on cranial ultrasonography. Graded 0-3: 1, subependymal haemorrhage; 
2, intraventricular haemorrhage; 3, parenchymal extension of intraventricular haemorrhage.

Parenchymal lesion. Evidence on cranial ultrasound of ventriculomegaly or cystic leucomalacia before discharge from unit.

\section{REFERENCES}

1 Cooke RWI. Annual audit of three year outcome in very low birth weight infants. Arch Dis Child 1993;63:295-8.

2 Powls A, Botting N, Marlow N et al. Motor impairment in children 12-13 years old with a birth weight of less than $1250 \mathrm{~g}$. Arch Dis Child Fetal Neonatal Ed 1995;72:F62-6.

3 Botting N, Powls A, Cooke RW, et al. Cognitive and educational outcome of very low birth weight children in early adolescence. Dev Med Child Neurol 1998;40:661-6.

4 Botting N, Powls A, Cooke RW, et al. Attention deficit hyperactivity disorders and other psychiatric outcomes in very low birth weight children at 12 years. J Child Psychol Psychiatry 1997;38:931-41

5 Powls A, Botting N, Cooke RW, et al. Growth impairment in very low birth weight children at 12 years: correlation with perinatal and outcome variables. Arch Dis Child Fetal Neonatal Ed 1996;75:F152-7.

6 Klebanov PK, Brooks-Gunn J, McCormick MC. School achievement and failure in very low birth weight children. J Dev Behav Pediatr 1994; 15:248-56.

7 Cooke RW. Growth impairment in the very preterm and cognitive and motor performance at 7 years. Arch Dis Child 2003;88:1-6.
8 Wechsler D. Wechsler intelligence scale for children, 3rd ed. London: The Psychological Corporation, Harcourt Brace and Co, 1992.

9 Henderson SE, Sugden DA. Movement ABC. London: The Psychological Corporation, Harcourt Brace and Co, 1992.

10 Bhutta AT, Cleves MA, Casey PH, et al. Cognitive and beahioural outcomes of school-aged children who were born preterm: a meta-analysis. JAMA 2002;288:728-37.

11 Cooke RW. Persistent arterial duct in the preterm infant. In: Anderson RH, Baker EJ, Macartney FJ, et al. Paediatric cardiology. London: Churchill Livingstone, 2002, chapter 25.

12 Knight DB. The treatment of patent ductus arteriosus in preterm infants. A review and overview of randomised trials. Semin Neonatol $2001 ; 6: 63-73$.

13 Klucknow M, Evans N. Low superior vena cava flow and intraventricular haemorrhage in preterm infants. Arch Dis Child Fetal Neonatal Ed 2000;82:F188-94.

14 Meek JH, Tyszczuk L, Elwell CE, et al. Low cerebral blood flow is a risk factor for severe intraventricular haemorrhage. Arch Dis Child Fetal Neonatal Ed 1999;81:F15-18.

15 Schmidt B, Davis P, Moddemann D, et al. Long term effects of indomethacin prophylaxis in extremely-low-birth-weight infants. N Engl J Med 2001;344:1966-72.

16 Abernethy L, Cooke RW, Foulder-Hughes L. Caudate and hippocampal volumes, intelligence and motor impairment in 7-year-old children who were born preterm. Pediatr Res 2004;55:884-93.

17 Abernethy L, Klafkowski G, Foulder-Hughes L, et al. Magnetic resonance imaging and T2 relaxometry of cerebral white matter and hippocampus in children born preterm. Pediatr Res 2003;54:868-74. 